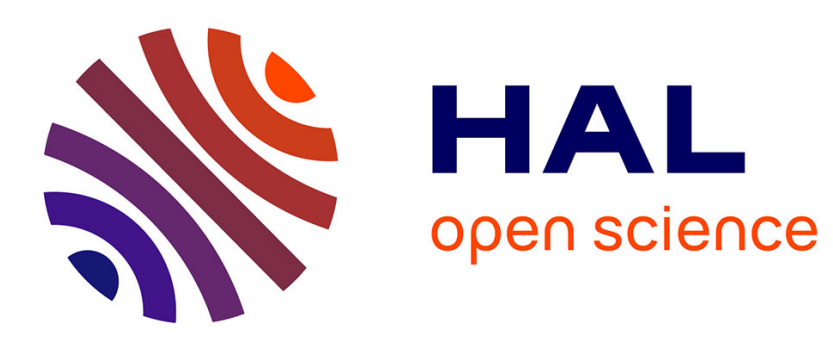

\title{
Influence of a multiaxial stress on the reversible and irreversible magnetic behaviour of a $3 \% \mathrm{Si}-\mathrm{Fe}$ alloy
}

Mahmoud Rekik, Olivier Hubert, Laurent Daniel

\section{To cite this version:}

Mahmoud Rekik, Olivier Hubert, Laurent Daniel. Influence of a multiaxial stress on the reversible and irreversible magnetic behaviour of a $3 \% \mathrm{Si}-\mathrm{Fe}$ alloy. International Journal of Applied Electromagnetics and Mechanics, 2014, 44 (3-4), pp.301-315. 10.3233/JAE-141793 . hal-01003012

\section{HAL Id: hal-01003012 \\ https://hal.science/hal-01003012}

Submitted on 8 Jun 2014

HAL is a multi-disciplinary open access archive for the deposit and dissemination of scientific research documents, whether they are published or not. The documents may come from teaching and research institutions in France or abroad, or from public or private research centers.
L'archive ouverte pluridisciplinaire HAL, est destinée au dépôt et à la diffusion de documents scientifiques de niveau recherche, publiés ou non, émanant des établissements d'enseignement et de recherche français ou étrangers, des laboratoires publics ou privés. 


\title{
Influence of a multiaxial stress on the reversible and irreversible magnetic behaviour of a $3 \% \mathrm{Si}-\mathrm{Fe}$ alloy
}

\author{
Mahmoud REKIK ${ }^{\mathrm{a}, 1}$, Olivier HUBERT ${ }^{\mathrm{a}}$ and Laurent DANIEL ${ }^{\mathrm{b}, \mathrm{c}}$ \\ ${ }^{a}$ LMT-Cachan, ENS Cachan, CNRS (UMR8535), UPMC, PRES Universud Paris \\ 61 avenue du Président Wilson, 94235 Cachan, France \\ ${ }^{\mathrm{b}}$ LGEP, SUPELEC, CNRS (UMR8507), UPMC, Univ Paris-Sud, \\ 11 rue Joliot-Curie, 91192 Gif sur Yvette, France \\ ${ }^{\mathrm{c}}$ School of Materials, University of Manchester, MI 7HS, Manchester, UK
}

\begin{abstract}
.
The research presented in this paper is motivated by the design of electrical devices submitted to mechanical stress. It aims at studying the magnetic behaviour of ferromagnetic materials submitted to biaxial stress. It deals with the evolution of the magnetic susceptibility, coercive field and magnetic losses with respect to stress, magnetic field level and frequency. These quantities are of primary importance in the design of rotors for high speed rotating machines.

The work is focused on the magnetic behaviour of a standard grade of Iron-Silicon alloy under the form of thin sheet. Non conventional experiments are performed on cross-shaped samples in order to apply biaxial stress representative of the loadings experienced by rotors of rotating machines. These experiments are performed on a multiaxial testing machine, ASTREE. The magnetic loading is applied using a single $\mathrm{U}$-yoke. The measurement of magnetic induction, magnetic field and strain is conducted by the means of needle-B sensor, H-coil sensors and Digital Image Correlation (DIC) respectively. Both anhysteretic and dissipative responses to magneto-mechanical loadings are considered. The results allow to identify the more critical stress configurations for this material.
\end{abstract}

Keywords. High speed rotating machines, biaxial stress, iron-silicon alloy, anhysteretic behaviour, dissipative behaviour, coercive field, power losses.

\section{Introduction}

Electrical parts of an aircraft represent approximately $35 \%$ of the mass of equipment in the electrical power chain. In addition, the electrical power to be released on new generation of aeronautics equipment is multiplied by 4 compared to conventional aircraft. This induces an exponential increase in the number, mass and size of electrical equipment. To achieve the objectives of weight reduction it is mandatory to find appropriate solutions to optimize electrical systems. One solution is to increase the power density

\footnotetext{
${ }^{1}$ Corresponding Author: M. REKIK; tel.: +33 1474077 62; email: rekik@1mt.ens-cachan.fr
} 
of generators. This requires higher rotation speed, leading to higher levels of centrifugal forces and stress in the rotor [1]. This speed contribution to stress comes in addition to other stress sources that can be inherited from forming and assembly processes (cutting, stacking, welding, ....).

The objective of this paper is to give experimental evidence of the influence of stress on the properties of electrical steels under biaxial stress for several frequency regimes. The magnetic material of interest in this study is a commercial non-oriented $3 \% \mathrm{Si}-\mathrm{Fe}$ from Arcelormittal delivered in $0.5 \mathrm{~mm}$ thick sheets. It is a standard material for rotating machines. A precise understanding of the complex magneto-mechanical coupling effects in this material is necessary in order to perform accurate magneto-mechanical structural analysis on electromagnetic devices. In section 2 the stress associated to centrifugal forces in a rotor is evaluated on a standard geometry for high speed rotating machine. In section 3 the experimental setup for multiaxial magneto-mechanical characterisation is described and the results are presented and discussed in sections 4 and 5.

\section{Multiaxial stress in rotating machines}

Modern technologies of wound rotor synchronous alternators for aeronautical applications involve higher and higher rotating speed and torque. High rotation speed creates high level of centrifugal forces. The first step of our analysis consists in estimating the stress experienced by a rotor under such high speed configurations. The analysis is limited here to the stress associated to centrifugal forces, but other sources of stress could be superimposed (e.g magnetic forces, torque..). A finite element modelling of a sheet metal rotor at angular velocity $\omega=\omega . \mathbf{e}_{z}$ has been performed using the finite element code ABAQUS $\subseteq$. Figure 2 shows the geometry of the chosen rotor.

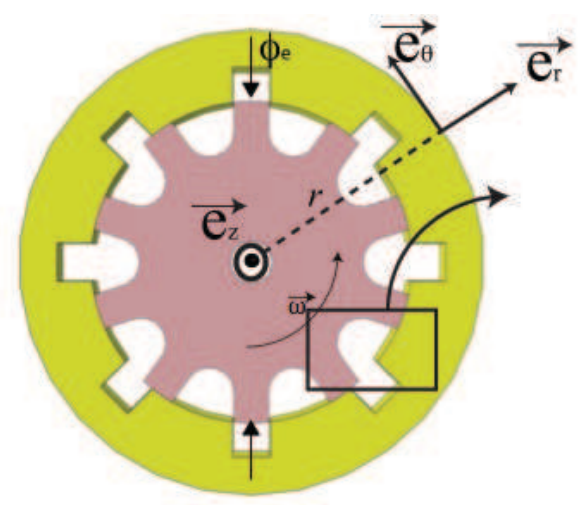

(a) Variable frequency generator

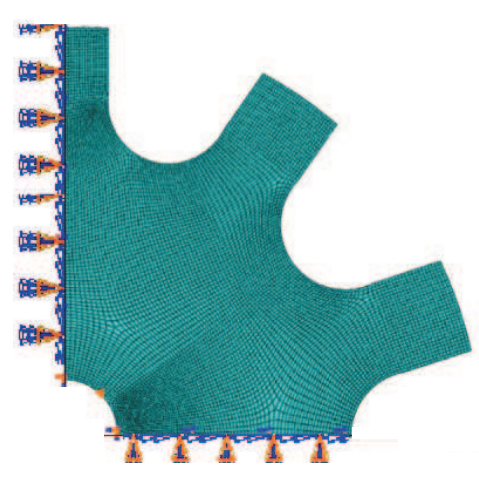

(b) Boundary conditions and mesh

Figure 1. Example of rotor/stator geometry of a high speed-rotating machine.

Volume forces are radial (Eq.(1)), $\rho$ is the mass density and $r$ the radial position.

$$
\mathbf{f}_{v}=\rho r \omega^{2} \mathbf{e}_{r}
$$


A quarter of the rotor is modelled. Figure 2 shows the mesh and the boundary conditions corresponding to the symmetry of the problem. Considering the small thickness of the sheet $(e=0.5 \mathrm{~mm})$ simulations are realized under plane stress assumptions. Local stress is calculated at each node of the mesh. The resulting stress tensor is under the form of Eq.(2) in cylindrical coordinates.

$$
\sigma=\left(\begin{array}{ll}
\sigma_{r r} & \tau_{r \theta} \\
\tau_{r \theta} & \sigma_{\theta \theta}
\end{array}\right)
$$

The calculated components of the stress tensor in the rotor are plotted in figures 2 and 3 for the angular velocity $\omega=4190 \mathrm{rad} / \mathrm{s}(40,000 \mathrm{rpm})$. The material properties have been taken isotropic in the sheet plane. The Young modulus is $E=191 \mathrm{GPa}$, the Poisson ratio $v=0.27$ and the mass density $\rho=7870 \mathrm{~kg} / \mathrm{m}^{3}$. The external radius of the rotor is $r_{\max }=38.0 \mathrm{~mm}$ and the internal radius is $r_{\min }=26.5 \mathrm{~mm}$.
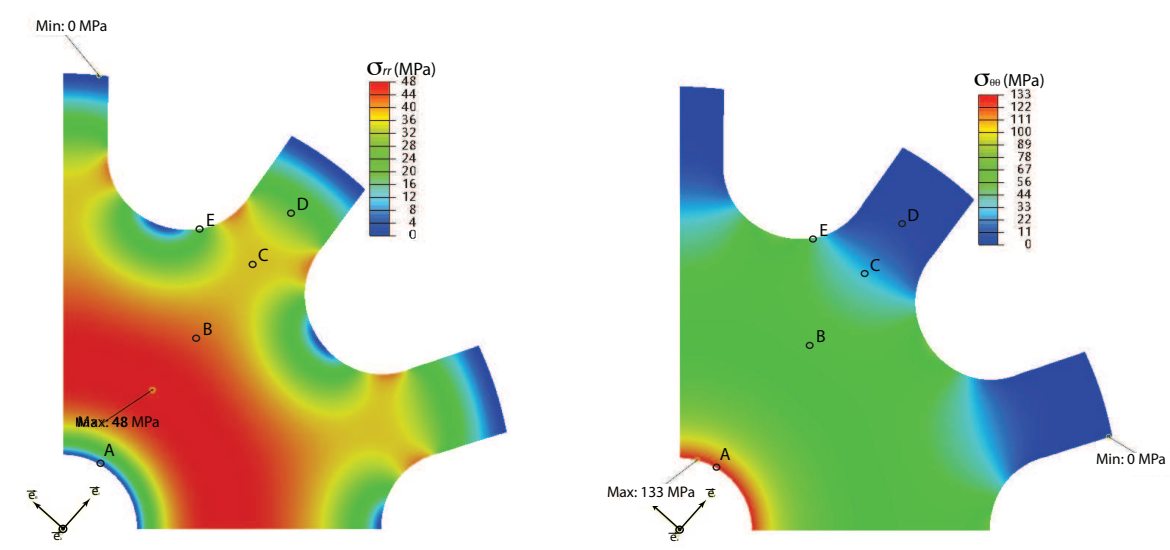

Figure 2. Calculated radial $\left(\sigma_{r r}\right)$ and tangential $\left(\sigma_{\theta \theta}\right)$ inertial stress distribution in the rotor.

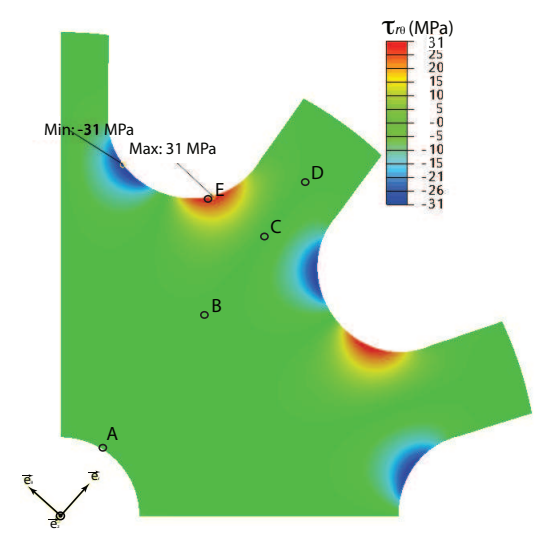

Figure 3. Calculated shear $\left(\tau_{r \theta}\right)$ inertial stress distribution in the rotor.

The stress tensor has been extracted and reported in Eq.(3) for a few points of interest A, 
$\mathrm{B}, \mathrm{C}, \mathrm{D}$ and $\mathrm{E}$ placed on the figures. The stress intensity is proportional to the angular velocity (values are reported for 40,000 rpm).

$$
\begin{aligned}
& \sigma_{A}=\left(\begin{array}{cc}
0 & 0 \\
0 & 133
\end{array}\right) \quad \sigma_{B}=\left(\begin{array}{cc}
46 & 0 \\
0 & 64
\end{array}\right) \quad \sigma_{C}=\left(\begin{array}{cc}
37 & -10 \\
-10 & 20
\end{array}\right) \\
& \sigma_{D}=\left(\begin{array}{cc}
20 & 0 \\
0 & 2
\end{array}\right) \quad \sigma_{E}=\left(\begin{array}{ll}
25 & 30 \\
30 & 46
\end{array}\right) \quad(\mathrm{MPa})
\end{aligned}
$$

$\sigma_{r r}$ and $\sigma_{\theta \theta}$ are positive on the entire structure. $\tau_{r \theta}$ is almost zero in most of the rotor except near the lower edges of the rotor teeth where stress concentration occurs (e.g points $\mathrm{C}$ and $\mathrm{E})$. Bi-tension $\left(\sigma_{r r}>0, \sigma_{\theta \theta}>0, \tau_{r \theta}=0\right)$ is the configuration the most encountered within the material (e.g points B and D). The orthoradial stress $\sigma_{\theta \theta}$ is often the dominant component of the stress tensor and can reach the double of $\sigma_{r r}$ in some areas (e.g point $\mathrm{E})$. In the teeth of the rotor, $\sigma_{\theta \theta}$ vanishes so that $\sigma_{r r}$ becomes the dominant component of the stress tensor (e.g point $\mathrm{D})$. The region near the axis of the rotor experiences high intensity tensile stress in the orthoradial direction (e.g point $\mathrm{A})$, and the external edges of the teeth are almost unstressed. The magnitude of stress is significant (of the order of a few tens of MPa) but remains far from the yield stress of the material (approximately $360 \mathrm{MPa}$ ). This finite element analysis illustrates the multiaxiality and the non-uniformity of stress within the rotor of a rotating machine.

\section{Experimental procedure}

The characterisation of magneto-mechanical behaviour is usually performed under uniaxial conditions $[2,3]$. The stress consists then of pure tension or compression applied along an axis parallel to the magnetic field. Such experiments cannot cover the complex interactions between stress and magnetic field under more general magnetomechanical loadings. Biaxial magneto-mechanical loading conditions can provide a deeper insight into magneto-elastic couplings but very few are reported in the literature $[4,5,6,7,8,9,10,11]$. The basic idea of biaxial tests is to perform a measurement of magnetic behaviour on a specimen loaded in tension-compression along two perpendicular directions. For that purpose, a cross-shaped specimen has been designed (figure 4a). Compression tests can be carried out if the specimen is designed to prevent buckling. This can be achieved thanks to a central core stuck to the specimen (figure $4 \mathrm{~b}$ ). Bakelite can be chosen for the core material. It is non-magnetic and non-conductive so that it does not disturb the magnetic measurement. The local stress tensor $\left(\sigma_{1}, \sigma_{2}\right)$ in the centre of the specimen can be calculated from the forces applied along the two loading axes $\left(F_{1}, F_{2}\right)$ according to an interacting matrix $\mathbf{K}$ with non-diagonal terms $(\sigma=\mathbf{K} . \mathbf{F})$. The terms of the matrix have been computed thanks to a finite element mechanical modelling of the specimen. The magnetic measurement area is a $30 \mathrm{~mm}$ diameter circle where both stress and magnetic field are fairly uniform (17.7\% [10]) (homogeneity of magnetic field has been verified for the same geometry in previous studies [12]).

Magnetic field and magnetic induction are measured thanks to a calibrated $H$-coil and a needle- $B$ sensor respectively. Strain field is obtained thanks to Digital Image Correlation (DIC) on the Bakelite side of the specimen. Figure 5 is a picture of the experimental 

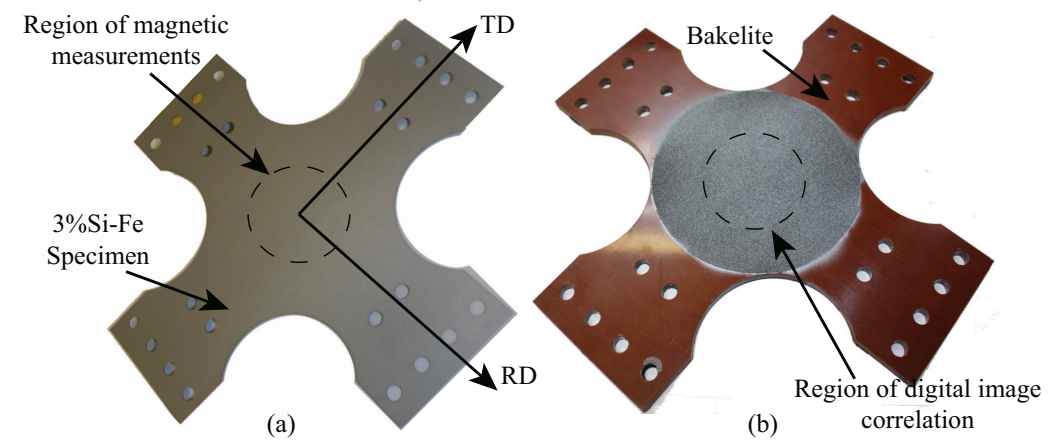

Figure 4. Experimental two-layer specimen: (a) specimen geometry; (b) core material: Bakelite and speckle used for DIC.

setup including the equipment used for DIC and figure 6 is a closer view on the specimen and the magnetic measurement apparatus put between the jacks of the hydraulic machine ASTREE.

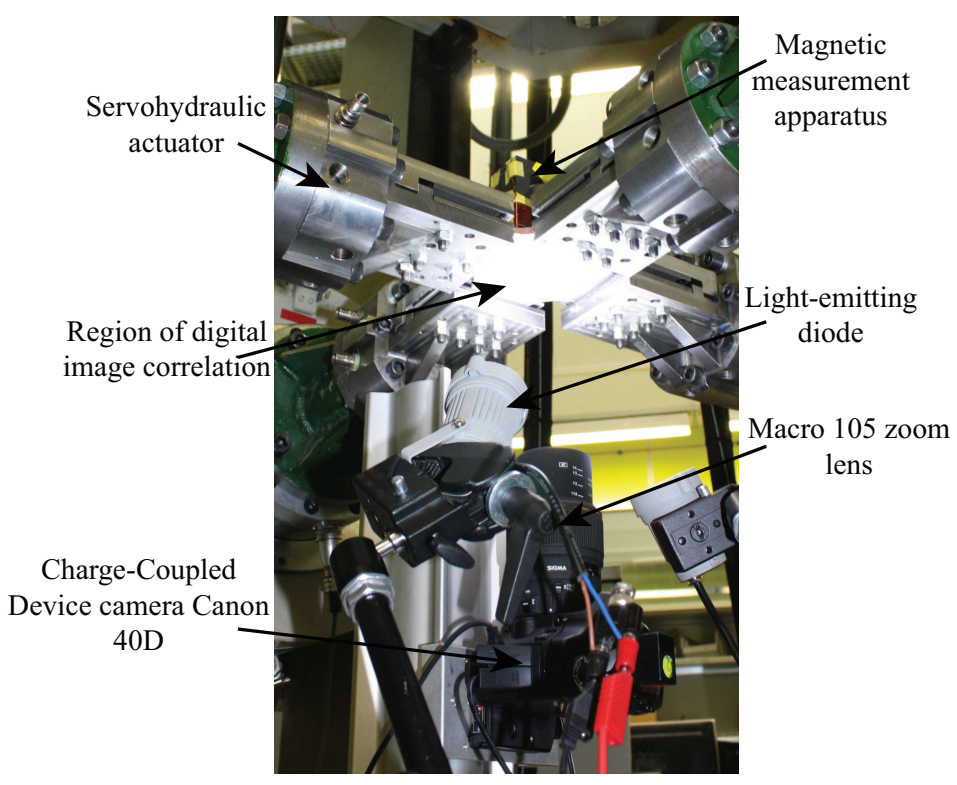

Figure 5. Experimental setup for biaxial testing.

The magnetic measurement procedure includes anhysteretic curves and hysteresis loops. Anhysteretic curves are constructed point after point by applying to the material a large amplitude alternating magnetic field superimposed on a dc magnetic field. The amplitude of the alternating field is slowly reduced to zero until only the dc field remains. 


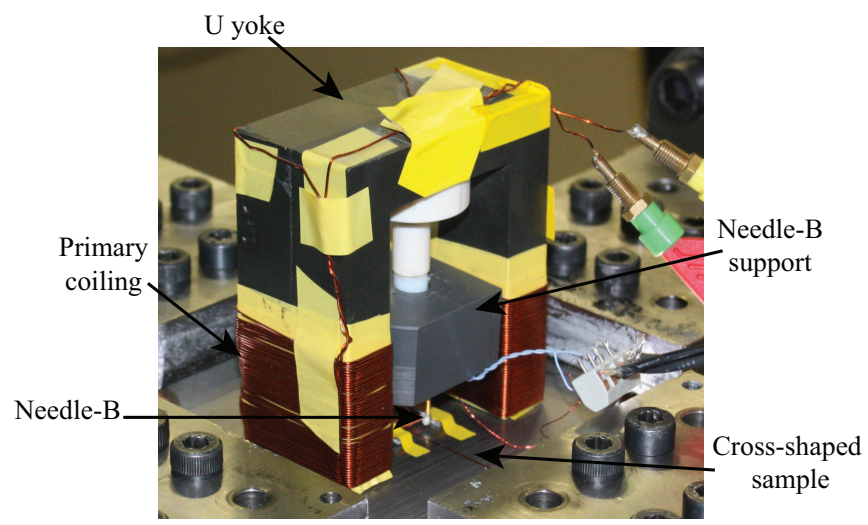

Figure 6. Magnetic measurements: U yoke and sensors.

This latter points defines the anhysteretic response corresponding to the applied dc field (figure 7). The material is demagnetised after each measurement of anhysteretic point to eliminate any influence of loading history in the measurement.

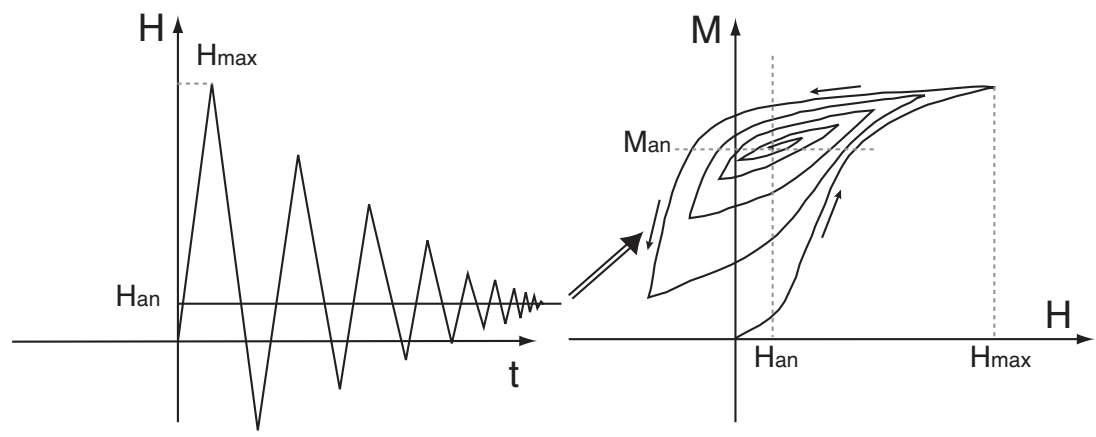

Figure 7. Principle of measurement for one point of the anhysteretic curve.

The measurement of hysteresis loops has been carried out at frequency of $\{f\}=\{1$, $5,10,50,100,200,400,800\} \mathrm{Hz}$. A triangular form of the current $i(t)$ has been used. The corresponding evolution of the measured magnetic field $H(t)$ is plotted in figure 8a and the magnetic induction $B(t)$ in figure $8 \mathrm{~b}$. The magnetic field is slighty distorted and remains fairly triangular while the magnetic induction is strongly distorted due to the non linearity of the magnetic behaviour.

The results can then be extracted in the form of magnetic induction curves $B(H)$ or magnetization curves $M(H)$ (figure 9) (using $B=\mu_{0}(H+M)$ ). The remnant induction $B_{r}$, the coercive field $H_{c}$ and the power losses $P$ can be calculated from the hysteresis loops. For a given value of the magnetic field $H$ and associated magnetization $M$ the secant susceptibility can also be defined : $\chi=M / H$.

For technical reasons related to the current command and non simultaneity f.e.m. measurement and integration, maximal induction can be subjected to a decrease at high frequencies $(>400 \mathrm{~Hz})$. We define a correction factor $r^{i}\left(0.9<r^{i}<1\right)$ which represents the ratio between the maximum induction $B_{\max }^{i}$ at given frequency $(i \in\{f\})$ and the maximum induction $B_{\max }^{1}$ at $f=1 \mathrm{~Hz}$ (Eq.(4)). 


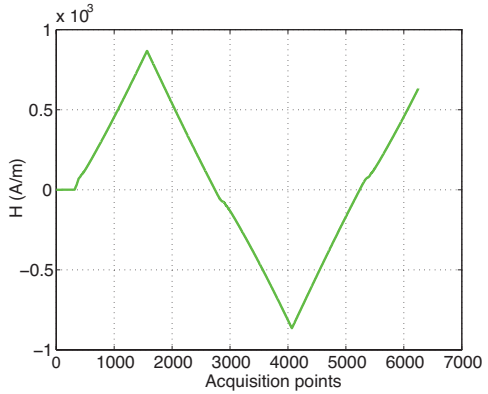

(a) Magnetic field $H$

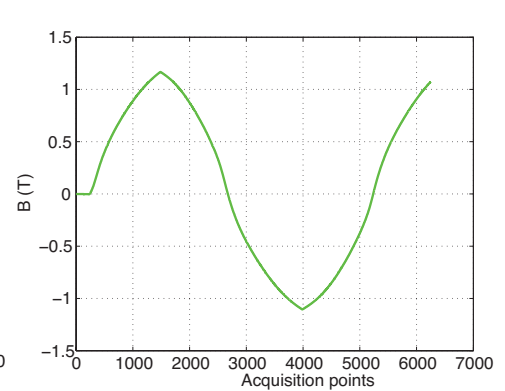

(b) Magnetic induction $B$

Figure 8. Magnetic measurement output as a function of time.

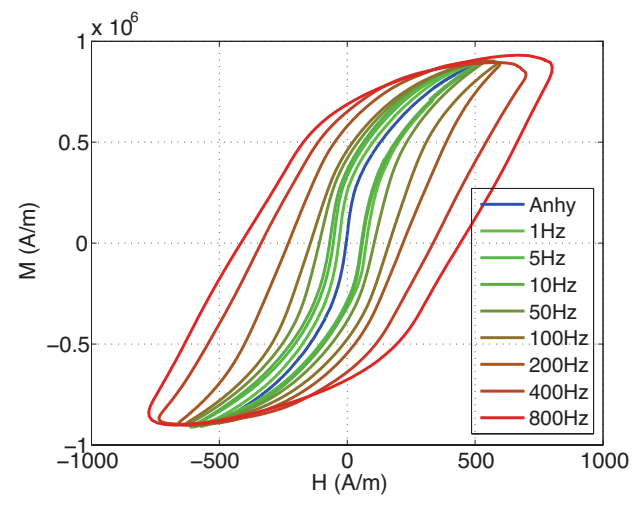

Figure 9. Examples of hysteresis loops for $\sigma_{1}=50 \mathrm{MPa}$ and $\sigma_{2}=-50 \mathrm{MPa}$ at different frequencies $\left(B_{\max } \simeq 1.1 T\right)$.

$$
r^{i}=\frac{B_{\text {max }}^{i}}{B_{\text {max }}^{1}}
$$

This correction allows the comparison of hysteretic parameters $\left(H_{c}, B_{r}, P\right)$ at a given value of $B_{\max }$. A linear correction is applied so that (Eq.(5)):

$$
\begin{aligned}
& \left(H_{c}^{i}\right)_{\text {corrected }}=\frac{H_{c}^{i}}{r^{i}} \\
& \left(B_{r}^{i}\right)_{\text {corrected }}=\frac{B_{r}^{i}}{r^{i}} \\
& \left(P^{i}\right)_{\text {corrected }}=\frac{P^{i}}{r^{i}}
\end{aligned}
$$

The measurement procedure is applied for several biaxial mechanical loadings $\left(\sigma_{1}, \sigma_{2}\right)$. The magnetic field is applied in the direction parallel to axis 1.41 biaxial loading configurations have been tested, for stress level varying from -100 MPa to +100 MPa. These stress states are plotted in the $\left(\sigma_{1}, \sigma_{2}\right)$ plane in figure 10 . 


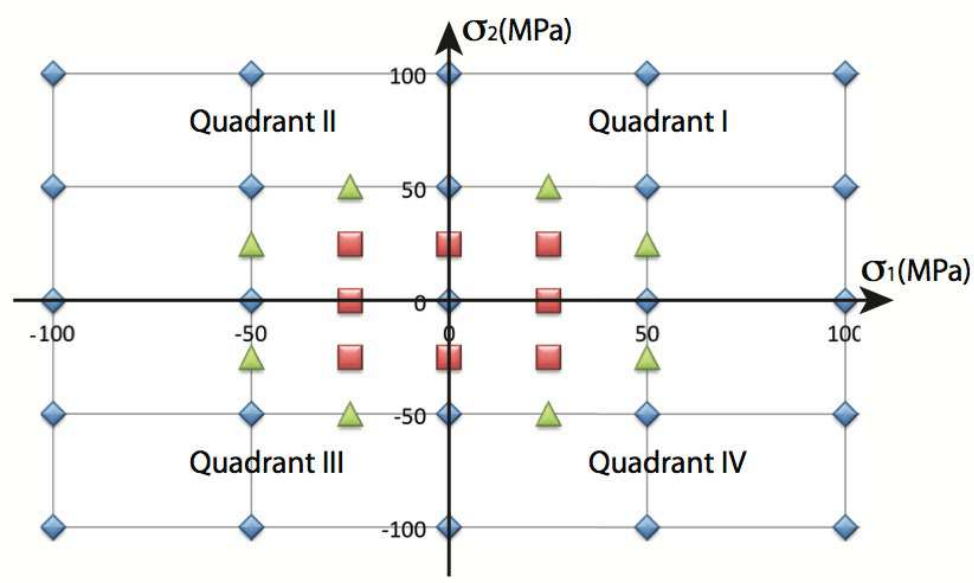

Figure 10. Experimental points in the $\left(\sigma_{1}, \sigma_{2}\right)$ plane and definition of 4 quadrants.

\section{Results}

\subsection{Anhysteretic magnetization curves}

Magnetization curves under uniaxial stress are given in figure 11. Figure 11(a) shows the case of a parallel uniaxial stress for which the uniaxial stress is applied in the direction of the magnetic field. The stress configuration is then given by $\left(\sigma_{1}, 0\right)$. The compression progressively deteriorates the magnetic behaviour - the magnetic susceptibility decreases - while tension enhances the magnetic behaviour (for the stress levels reported here). In the case of a perpendicular uniaxial stress (figure 11(b)) for which the uniaxial stress is applied in the direction orthogonal to the magnetic field, the stress configuration is $\left(0, \sigma_{2}\right)$. The effect of stress is significantly reduced in that configuration. The magnetic behaviour is slightly deteriorated in the first stages of magnetization and then slightly enhanced after $1000 \mathrm{~A} / \mathrm{m}$. The effect of tension is stronger than the effect of compression for a given intensity of stress.

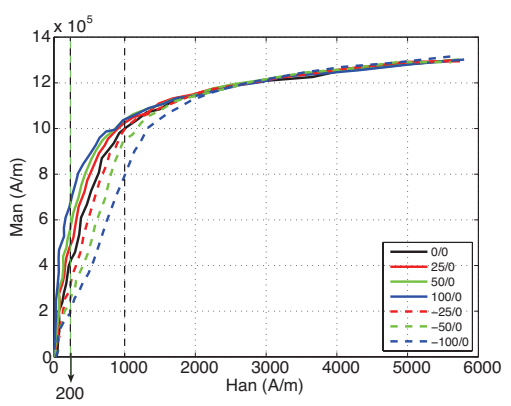

(a) Parallel uniaxial stress $\left(\sigma_{2}=0\right)$

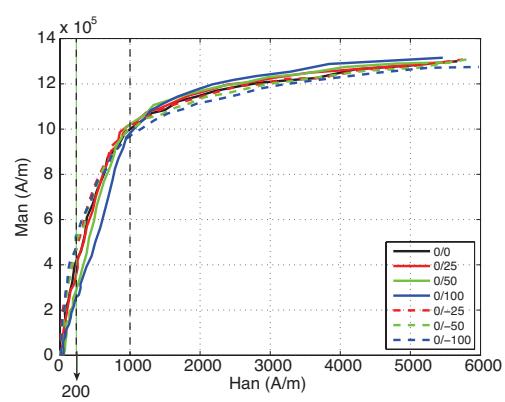

(b) Perpendicular uniaxial stress $\left(\sigma_{1}=0\right)$

Figure 11. Anhysteretic magnetization curves under uniaxial stress.

Magnetization curves under biaxial stress are given in figure 12. Under equibiaxial stress configuration (figure 12(a)), characterised by the condition $\sigma_{1}=\sigma_{2}$, negative stress 
components deteriorate the magnetic behaviour while positive stress components slightly enhance it. This effect is similar but lower in magnitude compared with parallel uniaxial stress configuration. Pure shear stress can also be applied $\left(\sigma_{1}=-\sigma_{2}\right)$. The effect of pure shear is hardly sensitive when the component of stress $\left(\sigma_{1}\right)$ parallel to the applied magnetic field is positive. But when $\sigma_{1}$ is negative, the deterioration of magnetic behaviour is very significant.

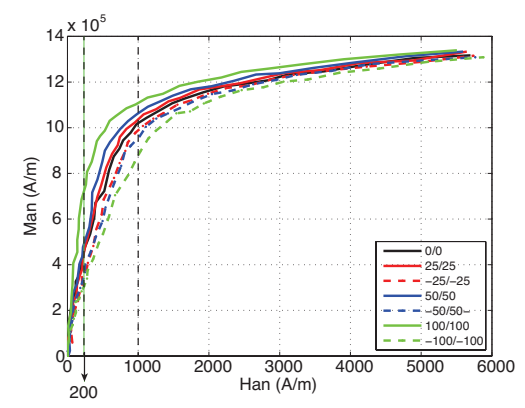

(a) Equibiaxial stress $\left(\sigma_{1}=\sigma_{2}\right)$

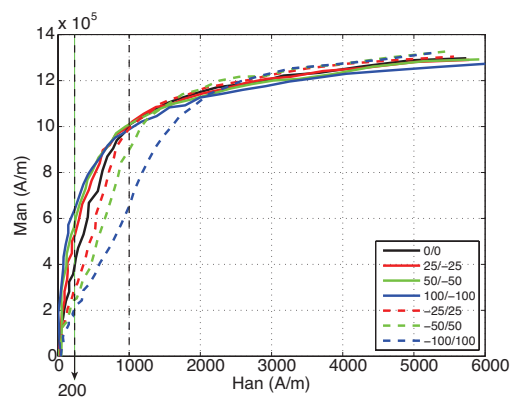

(b) Pure shear stress $\left(\sigma_{1}=-\sigma_{2}\right)$

Figure 12. Anhysteretic magnetization curves under biaxial stress.

\subsection{Secant susceptibility}

These results can also be analysed in terms of secant susceptibility $\chi$. Figure 13 plots the normalised secant susceptibility in the $\left(\sigma_{1}, \sigma_{2}\right)$ plane. The susceptibility under stress is normalised by the value of the susceptibility at the same magnetic field level under zero stress. Two levels of magnetic field (reported in figures 11 and 12) have been considered: $H=200 \mathrm{~A} / \mathrm{m}$ (figure 13(a)) and $H=1000 \mathrm{~A} / \mathrm{m}$ (figure 13(b)). The magnetic field is applied along direction 1 that is also the rolling direction (RD).

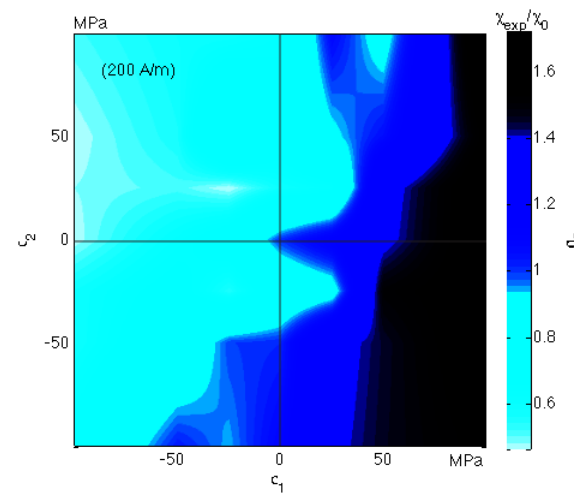

(a) $H=200 \mathrm{~A} / \mathrm{m}, \chi_{200}^{0}=2023$

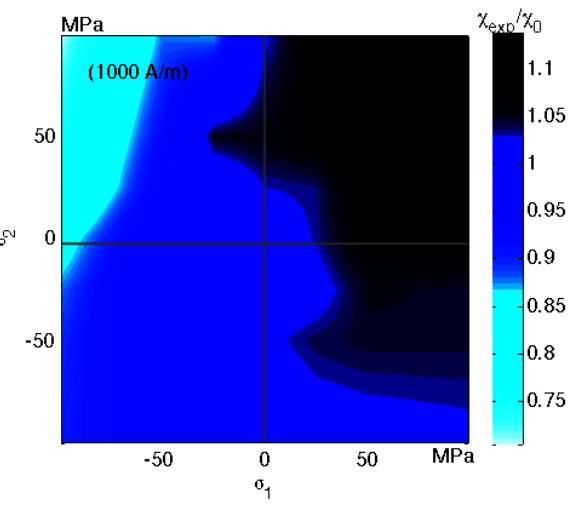

(b) $H=1000 \mathrm{~A} / \mathrm{m}, \chi_{1000}^{0}=976$

Figure 13. Normalised anhysteretic secant susceptibility under biaxial mechanical loading.

As already noticed in the previous section, a uniaxial tension along the magnetic field direction improves the susceptibility and a compression in the same direc- 
tion deteriorates the magnetic behaviour. When a uniaxial stress is applied in the direction perpendicular to the magnetic field, the effect is opposite and attenuated. The tension-compression asymmetry is very perceptible. Bi-tension increases the susceptibility $\left(\chi_{200}^{100,100}=1.6\right)$, while bi-compression decreases it $\left(\chi_{200}^{-100,-100}=0.7\right)$. The lowest values of $\chi$ are obtained in the second quadrant of the graph, corresponding to shear configurations. A compression along the magnetic field direction always tends to deteriorate the magnetic behaviour, whatever the value of $\sigma_{2}$. It can also be noticed that a bi-tension tends to slightly increase the magnetic susceptibility $\chi$. The effects of stress are less sensitive at high levels of magnetic field since the material is closer to saturation.

\subsection{Coercive field}

The variations $\Delta^{r} H_{c}$ of the coercive field $H_{c}$ under biaxial loading are given in figure 14 . These variations are calculated with respect to the unstressed configuration according to Eq.(6).

$$
\Delta^{r} H_{c}=100 \times \frac{H_{c}\left(\sigma_{1}, \sigma_{2}\right)-H_{c}(0,0)}{H_{c}(0,0)}=100 \times \frac{\Delta H_{c}}{H_{c}^{0}}
$$

It can be noticed that the variation $\Delta^{r} H_{c}$ is symmetric with respect to the line $\sigma_{1}=$ $-\sigma_{2}$. This variation is attenuated with increasing frequency. A strong similarity can be observed between the plots at low frequency $(f<200 \mathrm{~Hz})$. Under such frequencies the region of minimum coercive field is the fourth quadrant $\left(\sigma_{1}>0, \sigma_{2}<0\right)$ corresponding to shear stress with positive stress component along the magnetic field direction. Along the line of pure shear stress $\left(\sigma_{1}=-\sigma_{2}\right)$ in the second quadrant $\left(\sigma_{1}<0, \sigma_{2}>0\right)$, there is a strong increase of $H_{c}$ when the intensity of the stress is getting higher. This leads to the conclusion that shear stress has a very strong effect on the coercive field when the component of stress along the magnetic field direction is negative, but negligible otherwise. At frequencies of $400 \mathrm{~Hz}$ and higher, effect of stress on the coercive field is changing. The variations observed are lower. The area of minimum effect of stress becomes the equibiaxial region close to the line $\sigma_{1}=\sigma_{2}$. The maximum coercive field values are still observed for shear stress but now in the fourth quadrant, for positive $\sigma_{1}$. At $400 \mathrm{~Hz}$, a symmetry with respect to the line $\sigma_{1}=\sigma_{2}$ is observed in addition to the symmetry with respect to the line $\sigma_{1}=-\sigma_{2}$.

\subsection{Power losses}

The variations $\Delta^{r} P$ of the power losses $P$ under biaxial loading are given in figure 15 . These variations are calculated with respect to the unstressed configuration according to Eq.(7).

$$
\Delta^{r} P=100 \times \frac{P\left(\sigma_{1}, \sigma_{2}\right)-P(0,0)}{P(0,0)}=100 \times \frac{\Delta P}{P^{0}}
$$

The power losses at $1 \mathrm{~Hz}$ are not presented in figure 15 because the calculated value are very low $\left(P_{\max } \simeq 0.025 \mathrm{~W} / \mathrm{kg}\right)$ and associated to large uncertainties. For frequencies below $10 \mathrm{~Hz}$ the effect of stress on power losses is slight. A bi-tension state tends to 


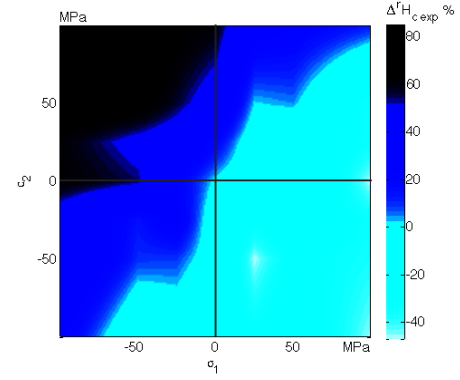

(a) $1 \mathrm{~Hz}$

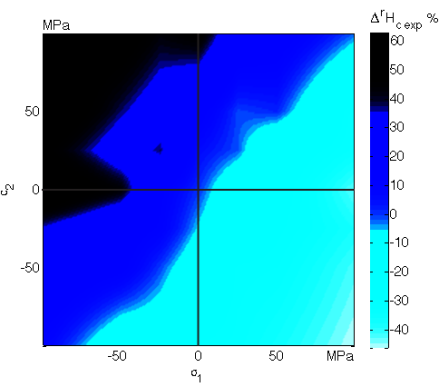

(c) $10 \mathrm{~Hz}$

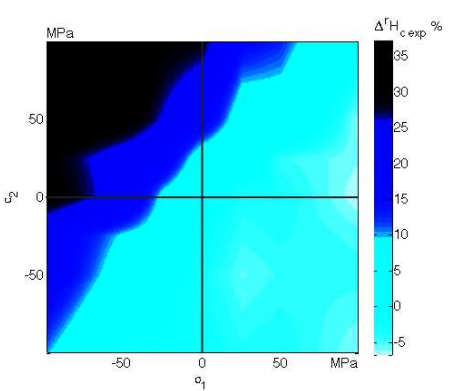

(e) $100 \mathrm{~Hz}$

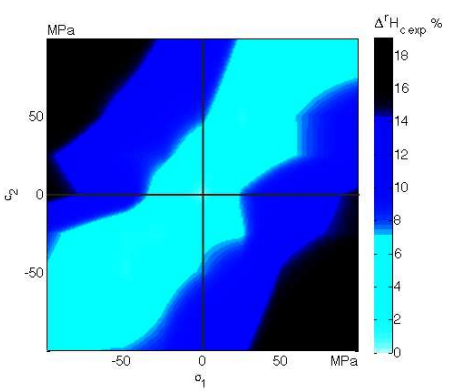

(g) $400 \mathrm{~Hz}$

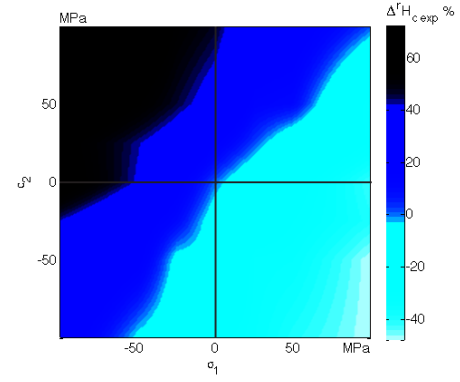

(b) $5 \mathrm{~Hz}$

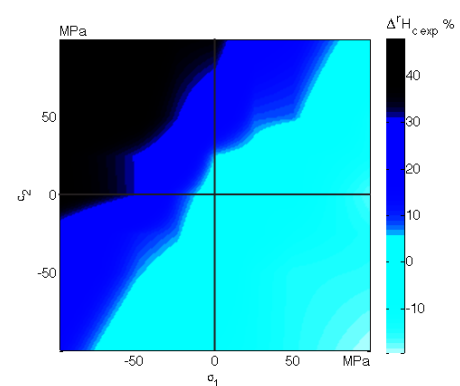

(d) $50 \mathrm{~Hz}$

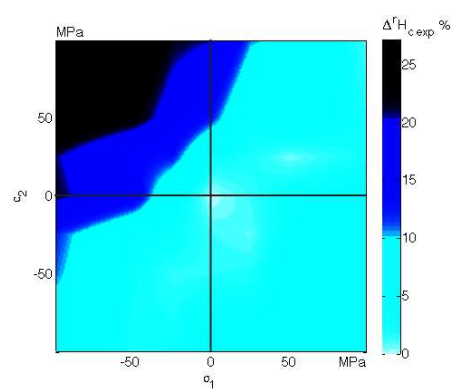

(f) $200 \mathrm{~Hz}$

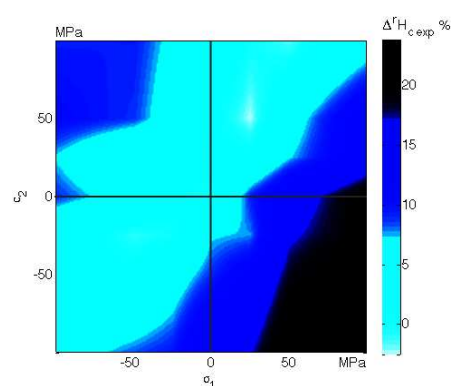

(h) $800 \mathrm{~Hz}$

Figure 14. Variations $\Delta^{r} H_{c}$ of coercive field under biaxial stress at different frequencies. 


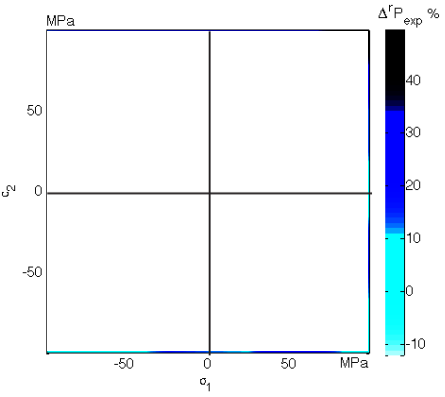

(a) $1 \mathrm{~Hz}$

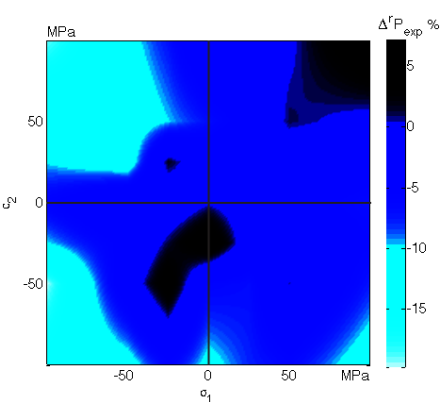

(c) $10 \mathrm{~Hz}$

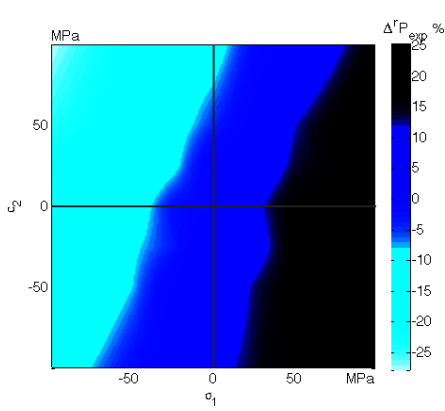

(e) $100 \mathrm{~Hz}$

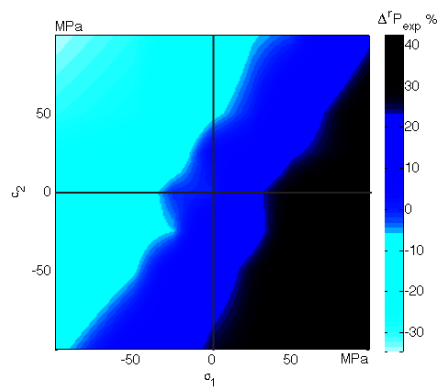

(g) $400 \mathrm{~Hz}$

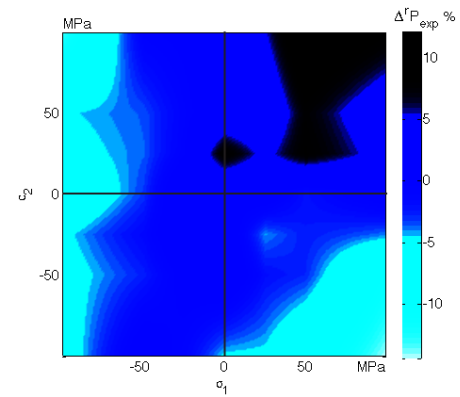

(b) $5 \mathrm{~Hz}$

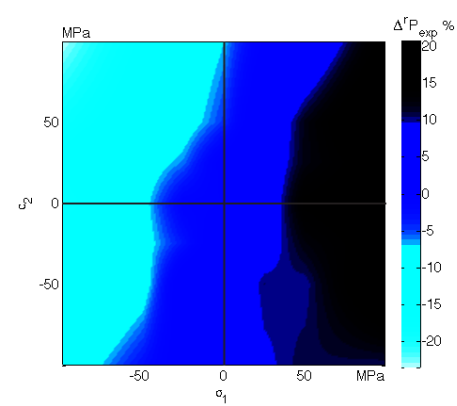

(d) $50 \mathrm{~Hz}$

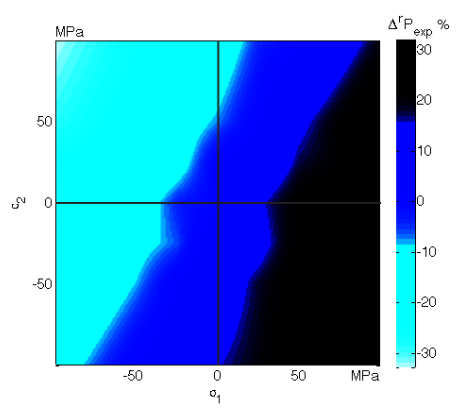

(f) $200 \mathrm{~Hz}$

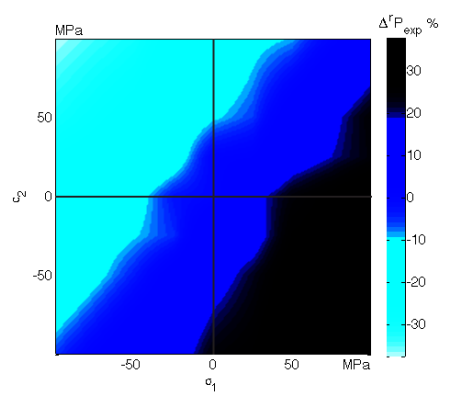

(h) $800 \mathrm{~Hz}$

Figure 15. Variations $\Delta^{r} P$ of power losses under biaxial stress at different frequencies. 
increase the magnetic power losses (by approximately $5 \%$ for $\sigma_{1}=\sigma_{2}=100 \mathrm{MPa}$ ). The highest power losses are obtained along the equibiaxial line $\sigma_{1}=\sigma_{2}$. For frequencies of $50 \mathrm{~Hz}$ and higher, the trends observed in the figures are very different. The highest power losses are now obtained for high positive $\sigma_{1}$. At $50 \mathrm{~Hz}$ this effect is almost independant of $\sigma_{2}$ but when the frequency increases the role of $\sigma_{2}$ becomes more significant. A negative $\sigma_{2}$ tends to increase the power losses. At high frequencies the higher power losses are obtained under shear stress with positive $\sigma_{1}$ (fourth quadrant). The iso-values of power losses are almost vertical lines at $50 \mathrm{~Hz}$ but the slope - positive - is decreasing apparently towards a slope of 1 as the frequency gets higher. The lower power losses are obtained for shear stress with negative $\sigma_{1}$ (second quadrant).

\section{Discussion}

\subsection{Secant susceptibility}

The results obtained in this work can be compared with the results obtained by Hubert [12] with a similar test rig on $0.5 \mathrm{~mm}$ thick sheets of an Iron-Cobalt alloy (49\%Co$49 \% \mathrm{Fe}-2 \% \mathrm{~V})$. The anhysteretic secant susceptibility was measured at $250 \mathrm{~A} / \mathrm{m}$ under biaxial stress loadings with maximum magnitude of $60 \mathrm{MPa}$ for each component. The results are plotted in figure 16(a) and the results of the present study are recalled in figure 16(b).

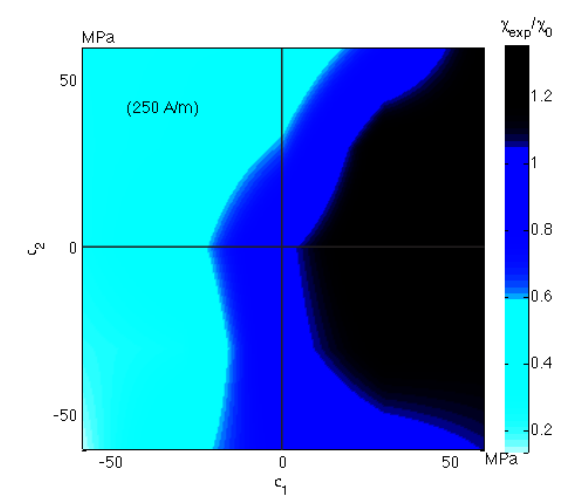

(a) Iron-Cobalt alloy [12], $H=250 \mathrm{~A} / \mathrm{m}$, $\chi_{250}^{0}=5000$

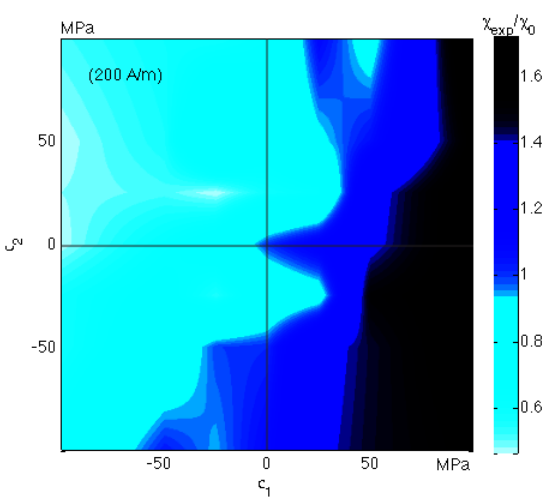

(b) Iron-Silicon alloy [this study], $H=200 \mathrm{~A} / \mathrm{m}$, $\chi_{200}^{0}=2023$

Figure 16. Normalised anhysteretic secant susceptibility under biaxial mechanical loading.

The tension-compression asymmetry is easily perceptible on both graphs. The predominant role of the stress component along the magnetic field is also a common feature of the two figures. But while the degradation under compressive parallel stress is dramatic for Iron-Cobalt it is moderate for the Iron-Silicon alloy. The increase of magnetic susceptibility under tensile stress is moderate for Iron-Cobalt whereas it is spectacular for the Iron-Silicon alloy. The iso-values of susceptibility are mainly directed in a vertical direction but this comparison highlights the complexity of the effects of stress on magnetic behaviour and notably their great dependency to the considered material. 


\subsection{Coercive field}

The results obtained in this work can be compared with the results obtained by Pearson $e t$ al. [13] at medium frequency $(50 \mathrm{~Hz})$ on $1 \mathrm{~mm}$ thick sheets of pure Iron. The variations of coercive field were measured under biaxial stress loadings with maximum magnitude of $30 \mathrm{MPa}$ for each component. The results are plotted in figure 17(a) and the results of the present study are recalled in figure 17(b). The main trends seem similar on both figures.

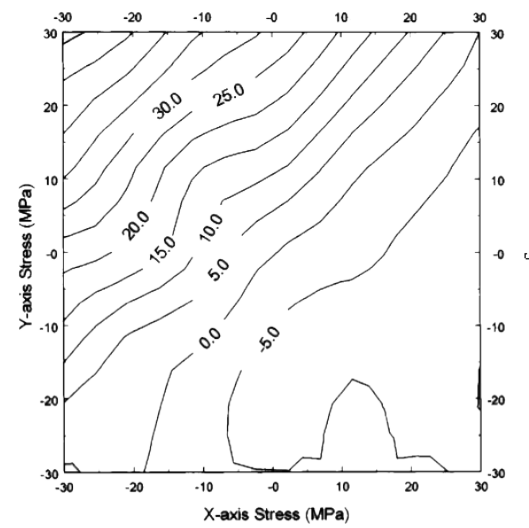

(a) Pure Iron [13]

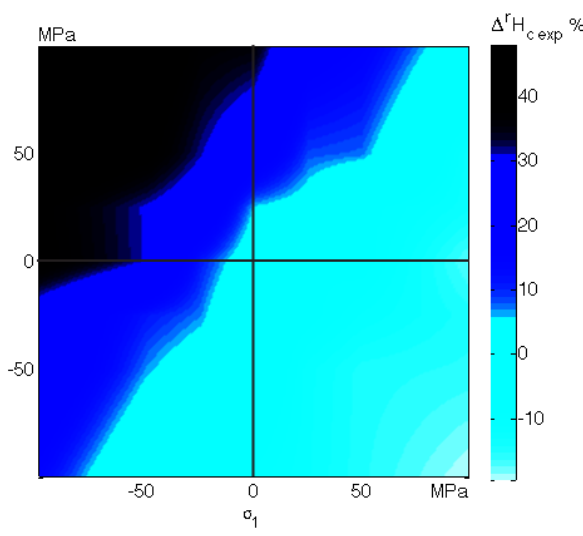

(b) Iron-Silicon alloy [this study]

Figure 17. Variations $\Delta^{r} H_{c}$ of coercive field under biaxial stress at $f=50 \mathrm{~Hz}$.

It is also interesting to look at the influence of frequency. To highlight the interaction of stress with frequency, the coercive field $H_{c}$ under pure shear loading as a function of the shear stress intensity $\tau\left(\tau=\frac{1}{2}\left(\sigma_{1}-\sigma_{2}\right)\right)$ is plotted in figure 18 at different frequencies. Indeed it was observed on the hysteresis loops that shear stress configurations have a strong effect on the coercive field, and that the pure shear line $\left(\sigma_{1}=-\sigma_{2}\right)$ was an axis of symmetry in figure 14 .

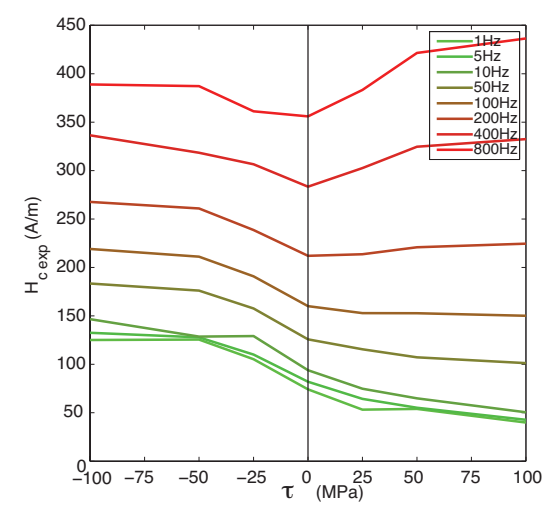

Figure 18. Coercive field under pure shear stress $\left(\sigma_{1}=-\sigma_{2}\right)$ as a function of shear stress intensity $\tau=\frac{1}{2}\left(\sigma_{1}-\sigma_{2}\right)$ at different frequencies. 
It is evident from figure 18 that the effect of stress and frequency are coupled. At low frequency an increasing shear stress tends to decrease the coercive field while it tends to increase the coercive field at high frequency. Again this observation points out the complexity of the effects of stress on magnetic behaviour.

\subsection{Power losses}

The same analysis on frequency effects can be made on power losses. Figure 19 shows the measured power losses per cycle $P / f$ under pure shear loading as a function of the shear stress intensity $\tau\left(\tau=\frac{1}{2}\left(\sigma_{1}-\sigma_{2}\right)\right)$ at different frequencies.

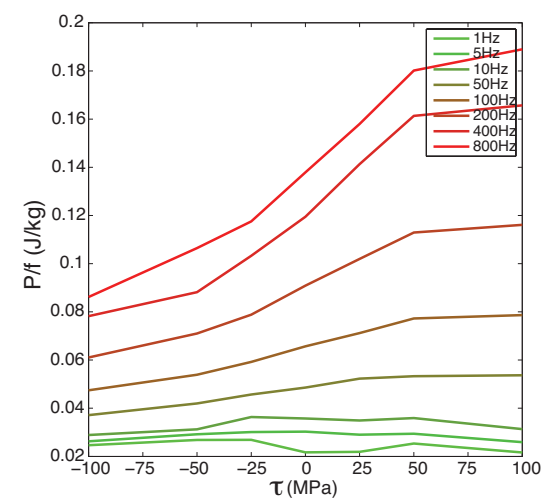

Figure 19. Power losses per cycle under pure shear stress $\left(\sigma_{1}=-\sigma_{2}\right)$ as a function of shear stress intensity $\tau=\frac{1}{2}\left(\sigma_{1}-\sigma_{2}\right)$ at different frequencies.

Eddy currents induced in the specimen cause significant losses. Above $100 \mathrm{~Hz}$, flux is mainly concentrated at the surface due to the skin effect, resulting in an increase of eddy current losses. On the other hand the change in permeability due to stress also affects the skin depth.

Another important issue confirming the interpretation is that the slope of iso-values of $P$ (figure 15) in the eigen stress frame is increasing with frequency, aligning with the slope of iso-values of permeability.

\section{Conclusion}

An experimental setup dedicated to observe the influence of biaxial stress and frequency on the magnetic behaviour of thin soft magnetic materials was described.

The obtained results for iron-silicon are consistent with the results published in previous work concerning susceptibility and coercive field. Experimental results of power losses evolution with biaxial stress and frequency provide a new contribution to the knowledge in the field.

All results confirm the pre-dominant role of the stress component along the magnetic field axis. Nevertheless the change of magnetic quantities with stress does not seem to follow the same rule depending on the magnetic quantity considered. On the other hand coercive field and power losses exhibit both a coupling with frequency strongly depen- 
dent to the stress. Accurate design of high speed rotating machines requires consequently numerical models taking into account these effects. Such numerical models do not exist today.

Multiscale phenomenological approaches are an interesting solutions [14,15]. But they refer to anhysteretic behaviour which is a strong limitation. This should be overcome.

\section{Acknowledgements}

The authors wish to thank Arcelormittal for providing the material and 3MT FUI-OSEO Project for the financial support.

\section{References}

[1] L. Bernard et al., Effect of stress on switched reluctance motors: a magneto-elastic finite-element approach based on multiscale constitutive laws, IEEE Trans. Magn., 47(9) : 2171-2178, 2011.

[2] R.M. Bozorth, Ferromagnetism, Norstand Company, NY, 1951.

[3] B.D. Cullity, Introduction to Magnetic Materials, Addison-Wesley, 1972.

[4] M.J. Sablik et al., Modeling biaxial stress effects on magnetic hysteresis in steel with the field and stress axes noncoaxial, J. Appl. Phys., 85 : 4391, 1999.

[5] C.S. Schneider et al., Biaxial magnetoelasticity in steels, J. Appl. Phys., 53 : 8136, 1982.

[6] K. Kashiwaya, Magnetoelastic effect of 3.25\% Si-Fe single crystal under low magnetic field, J. Appl. Phys., 30 : 2932, 1991.

[7] M.J. Sablik et al., Micromagnetic model for the influence of biaxial stress on hysteretic magnetic properties, J. Appl. Phys., 75 : 5673, 1994.

[8] M.J. Sablik et al., The effects of biaxial stress on Barkhausen noise signals when the magnetic field is noncoaxial with the stress axes, J. Appl.Phys., 84 : 6239, 1998.

[9] J. Pearson et al., Biaxial stress effects on the magnetic properties of pure iron, IEEE Trans. Magn., 36 : 3599, 2000.

[10] V. Maurel et al., Setup to test biaxial stress effect on magnetomechanic coupling, J. Appl. Phys., 93 : 7115, 2003.

[11] O. Hubert et al., A new experimental set-up for the characterization of magneto-mechanical behaviour of materials submitted to biaxial stresses. Application to FeCo alloys, Przeglad Elektrotechniczny, 81 : $19,2005$.

[12] O. Hubert, Influence of biaxial stresses on the magnetic behavior of an iron-cobalt sheet experiments and modelling, Przeglad Elektrotechniczny, 83(4) : 70, 2007.

[13] J. Pearson et al., Biaxial Stress Effects on the Magnetic Properties of Pure Iron, IEEE Trans. Magn., 36 : 3251-3253, 2000.

[14] L. Daniel et al., Reversible magneto-elastic behavior : A multiscale approach, J. Mech. Phys. Solids, 56 : 1018, 2008.

[15] O. Hubert, L. Daniel, Energetical and multiscale approaches for the definition of an equivalent stress for magneto-elastic couplings, J. Magn. Magn. Mater., 323(20) : 1766-1781, 2011. 